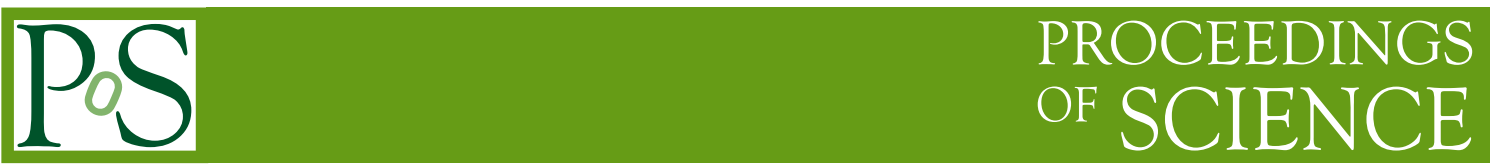

\title{
Recent top quark properties in CMS
}

\section{Agostino De lorio* for the CMS Collaboration}

Università degli Studi di Napoli "Federico II" e INFN sezione di Napoli

E-mail: adeioriodfisica.unina.it, deiorio@na.infn.it

The top quark is produced at the LHC in pair with its anti-particle via strong interactions and singly via electroweak interactions. An accurate knowledge of its properties (mass, couplings, production cross section, decay branching fractions, etc.) can bring key information on fundamental interactions at the electroweak symmetry-breaking scale and beyond. Measurements of top quark properties using data collected by the CMS experiment are presented in this note. Among them, latest results on top quark mass, $\mathrm{t} \overline{\mathrm{t}}$ spin correlations, Yukawa coupling and single top quark spin asymmetry are the most relevant.

European Physical Society Conference on High Energy Physics - EPS-HEP2019 -

10-17 July, 2019

Ghent, Belgium

${ }^{*}$ Speaker. 


\section{Top quark properties}

The top quark is the heaviest particle known in Nature. It has $\frac{1}{2}$ spin, $\frac{2}{3} e$ electric charge and color charge. So the top quark participates to all the interactions. It has a natural mass: its Yukawa coupling to the vacuum expectation value is close to 1 . For this reason it has a privileged relation with the Higgs boson and it could also have a role in the electroweak (EW) simmetry breaking mechanism. The top quark decays before the hadronisation can occur: its lifetime is of order of $2 \times 10^{-25}$ seconds. So its angular properties are directly accessible through its decay products. The top quark decays weakly almost exclusively in a $\mathrm{W}$ boson and a $\mathrm{b}$ quark.

\section{Top quark mass}

The top quark physics is mainly driven by its large mass. The value of top quark mass significantly affects the prediction for many observables, either directly or via radiative corrections. It is a key input to electroweak precision fits and, together with the values of the Higgs boson mass and of the strong interactions coupling $\alpha_{S}$, it has direct implications on the Standard Model (SM) predictions for the stability of the EW vacuum. The value of the top quark mass has also cosmological consequences, e.g. on Universe lifetime.

Top quark mass is challenging both for experiments and theory. From the theory side, different definitions of the top quark mass are used. Top quark MonteCarlo (MC) mass refers to the value used in the parton shower codes. Top quark pole mass or $\overline{\mathrm{MS}}$-mass refer to the fixed-order theoretical predictions. In the on-shell (o.s.) and $\overline{\mathrm{MS}}$ schemes the renormalised bare top quark propagator $S^{R}(p)$ can then be expressed in terms of pole and $\overline{\mathrm{MS}}$ masses, respectively, as follows:

$$
S_{\mathrm{o} . \mathrm{s} .}^{R}(p)=\frac{i}{\not p-m_{\mathrm{pole}}}, \quad S_{\overline{\mathrm{MS}}}^{R}(p, \mu)=\frac{i}{\not p-m_{\overline{\mathrm{MS}}}(\mu)-(A-B) m_{\overline{\mathrm{MS}}}(\mu)}
$$

The relation between top quark pole $\left(m_{\mathrm{t}}^{\text {pole }}\right)$ and $\overline{\mathrm{MS}}\left(m_{\mathrm{t}}\left(m_{\mathrm{t}}\right)\right)$ masses is calculated up to four loops in and reads:

$$
\begin{aligned}
m_{t}^{\text {pole }} & =\bar{m}_{t}\left(\bar{m}_{t}\right)\left[1+0.4244 \alpha_{S}+0.8345 \alpha_{S}^{2}+2.375 \alpha_{S}^{3}+(8.615 \pm 0.017) \alpha_{S}^{4}+O\left(\alpha_{S}^{5}\right)\right] \\
& =[163.508+7.529+1.606+0.496+(0.195 \pm 0.0004)] \mathrm{GeV}
\end{aligned}
$$

For a detailed discussion the reader could see Ref. [1]. The top quark mass can be measured both directly by fitting observables depending on top quark mass and indirectly by studying the property that depend on the top quark pole mass. The top quark mass measurement can be performed in many channels by exploiting many experimental observables and this allows to combine the different results to improve the precision.

\subsection{Direct top quark mass in $\ell+$ jets final state}

Top quark mass can be measured in $t \bar{t}$ events that decay in $\ell+$ jets final state [2]. The selection requires 1 high $p_{\mathrm{T}}$ isolated e/ $\mu$, at least 4 jets, two of them are required to be originated by a $\mathrm{b}$ quark. The full event reconstruction, necessary to access the invariant mass of the two top quarks, is done via a kinematic fit with the $\mathrm{W}$ boson mass constraint. The goodness of fit coming out from 
this procedure is used to weight the distributions of the kinematic variables. Then an ideogram method is applied to estimate the jet energy scale factor necessary to reduce the impact of the jet energy calibration uncertainty on the final measurement. Figure 1(a) shows the top quark mass distribution before, (a), and after the application of the weight, (b).

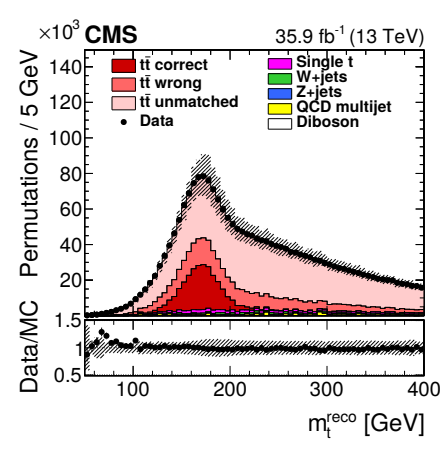

(a)

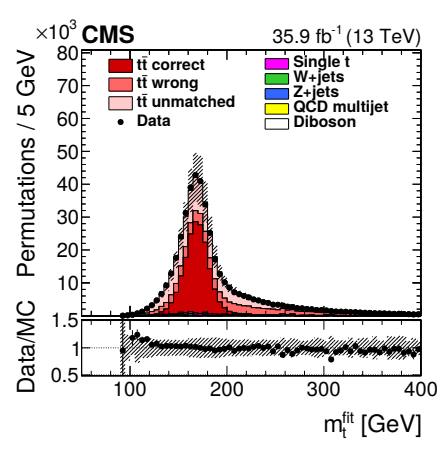

(b)

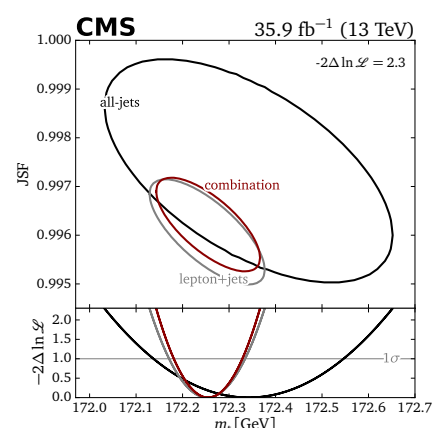

(c)

Figure 1: The top mass distribution before (a) and after the application of the weights (b) [2] and the shapes of the likelihood in the semi-leptonic, all-hadronic and combined cases (c) [3].

A simultaneous fit is performed in order to extract the top quark mass and the jet energy scale factor values obtaining:

$$
\begin{aligned}
m_{\mathrm{t}} & =172.25 \pm 0.08(\text { stat }) \pm 0.62(\text { syst }) \\
J S F & =0.996 \pm 0.001(\text { stat }) \pm 0.008(\text { syst })
\end{aligned}
$$

\subsection{Direct top quark mass in all-hadronic final state}

The top quark mass can also be measured in $t \bar{t}$ events that decay in all hadronic final state [3]. The selection requires 6 jets, two of them coming from the hadronisation of a $b$ quark. The analysis strategy is identical to the one used in the case of $\ell+$ jets final state. The fit in this case obtains:

$$
\begin{aligned}
m_{\mathrm{t}} & =172.34 \pm 0.20(\text { stat }) \pm 0.70(\text { syst }) \\
J S F & =0.997 \pm 0.002(\text { stat }) \pm 0.007(\text { syst })
\end{aligned}
$$

Due to the use of the same analysis strategy, it's possible to combine the two analyses with a single likelihood and find a more precise value. Figure 1c shows the likelihoods functions for the semi-leptonic, all-hadronic and combined cases. The results of the combined case are:

$$
\begin{aligned}
m_{\mathrm{t}} & =172.26 \pm 0.07(\text { stat }) \pm 0.61(\text { syst }) \\
J S F & =0.996 \pm 0.001(\text { stat }) \pm 0.007(\text { syst })
\end{aligned}
$$

This is the first combined top quark mass extraction from the lepton+jets and all-hadronic channels through a single likelihood function. 


\subsection{Indirect top quark pole mass in di-lepton final state}

For the top quark pole mass measurement, the selection requires one opposite flavour lepton pair with opposite charge [4]. Electrons and muons coming from the $\tau$ decay are excluded. A loose kinematic fit is used for the event reconstruction but the top mass constraint is not used. This allows to solve for the pair of neutrinos and consequently reconstruct the $t \bar{t}$ system. The triple differential cross section in terms of the invariant mass and the pseudorapidity of the $\bar{t} \bar{t}$ system and of the number of extra jets not arising from the decay of a top quark is used to extract the top quark pole mass as well as $\alpha_{S}$ using several sets of parton distribution functions (PDF), as shown in Figure 2.
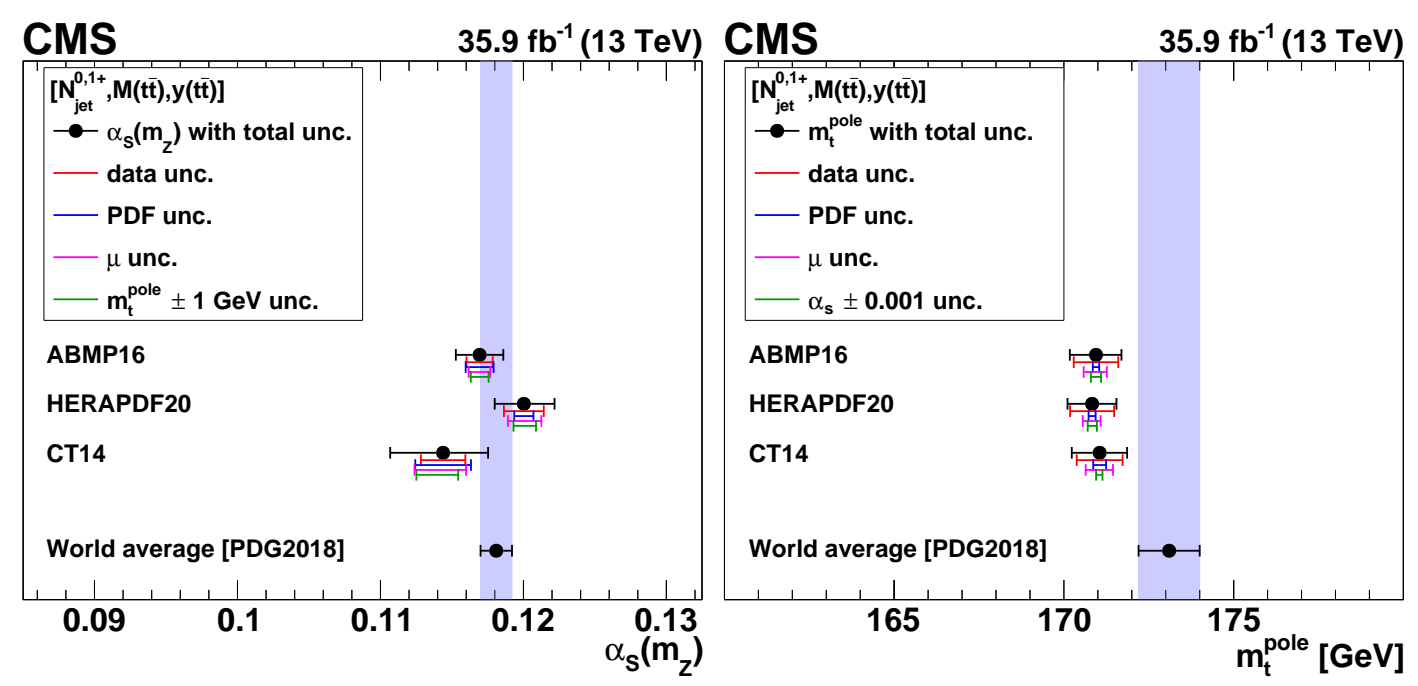

Figure 2: Top pole mass and $\alpha_{S}$ extraction at NLO for different PDF sets [4].

Furthermore, a simultaneous fit of the PDFs, $\alpha_{S}$, and top pole mass is performed at next-to-leading order (NLO), demonstrating that the new data have significant impact on the gluon PDF, and at the same time allow an accurate determination of $\alpha_{S}$ and $m_{\mathrm{t}}^{\text {pole }}$.

$$
\begin{aligned}
\alpha_{S}\left(m_{\mathrm{Z}}\right) & =0.1135 \pm 0.0016(\text { fit })_{-0.0004}^{+0.0002}(\text { model })_{-0.0001}^{+0.0008}(\text { param })_{-0.0005}^{+0.0011}(\text { scale }) \\
m_{\mathrm{t}}^{\text {pole }} & =170.5 \pm 0.7(\text { fit }) \pm 0.1(\text { model })_{-0.1}^{+0.0}(\text { param }) \pm 0.3(\text { scale }) \mathrm{GeV}
\end{aligned}
$$

\subsection{Direct/Indirect top quark pole mass in di-lepton final state}

Top quark MC mass and top quark pole mass are measured in $t \bar{t}$ events that decay in di-lepton final state by requiring two opposite flavour leptons with opposite charge [5]. A kinematic fit is used to fully reconstruct the event. To increase the sensitivity to the top quark mass, the minimum invariant mass of the lepton and the $b$ jet system is used. 12 regions are defined in terms of the number of $b$ jets and of extra jets present in the event. Finally, a simultaneous fit is performed to measure the cross section and the top quark MC mass:

$$
\begin{aligned}
& \sigma_{\mathrm{t}}=815 \pm 2(\text { stat }) \pm 29(\text { syst }) \pm 20(\text { lumi }) \mathrm{pb} \\
& m_{\mathrm{t}}^{\mathrm{MC}}=172.33 \pm 0.14(\text { stat })_{-0.72}^{+0.66}(\text { syst }) \mathrm{GeV}
\end{aligned}
$$

The cross section has a residual dependence on top quark MC mass and it is used to extract the value of the top quark mass in the $\overline{\mathrm{MS}}$ scheme for different PDF sets as reported in Table 1 . The 


\begin{tabular}{lc}
\hline PDF set & $m_{\mathrm{t}}^{\text {pole }}[\mathrm{GeV}]$ \\
\hline ABMP16 & $169.9 \pm 1.8_{-1.2}^{+0.8}$ \\
NNPDF3.1 & $173.2 \pm 1.9_{-1.3}^{+0.9}$ \\
CT14 & $173.7 \pm 2.0_{-1.4}^{+0.9}$ \\
MMHT14 & $173.6 \pm 1.9_{-1.4}^{+0.9}$ \\
\hline
\end{tabular}

Table 1: Value of the top quark mass in the $\overline{\mathrm{MS}}$ scheme for different PDF sets. The first uncertainty is due to the fit $+\mathrm{PDF}+\alpha_{S}$ contributes while the Figure 3: Indirect determination of $m_{\mathrm{t}}\left(m_{\mathrm{t}}\right)$ and $\alpha_{S}$ for difsecond is due to the scale variation. ferent PDF sets [5].

residual dependence on top MC mass allows also an indirect determination of $m_{\mathrm{t}}\left(m_{\mathrm{t}}\right)$ and $\alpha_{S}$ as shown in Figure 3.

\section{Top quark polarisation}

The top quark decay width is larger than the QCD hadronisation scale and of the spin decorrelation scale. This implies that, not only does the top quark decay before hadronisation occurs, but also its spin information is preserved in the angular distribution of its decay products. The top quarks and antiquarks that are produced at the Large Hadron Collider (LHC) via the strong interaction are mostly unpolarised at leading order. However, the spins of the top quarks and antiquarks are strongly correlated. Squared matrix element can be written as reported in Eq. 3.1 by factorizing decay density matrices $\rho$ and $\bar{\rho}$.

$$
\left|\mathscr{M}\left(\mathrm{q} \overline{\mathrm{q}} / g g \rightarrow \mathrm{t} \overline{\mathrm{t}} \rightarrow\left(\ell^{+} v b\right)\left(\ell^{-} \bar{v} \bar{b}\right)\right)\right|^{2} \sim \operatorname{Tr}[\rho R \bar{\rho}]
$$

The spin density matrix $R$ can be studied by using the double differential cross section in terms of the polarization angles of the top quark and antiquark:

$$
\frac{1}{\sigma} \frac{d \sigma}{d \cos \theta_{1}^{i} d \cos \theta_{2}^{j}}=\frac{1}{4}\left(1+B_{1}^{i} \cos \theta_{1}^{i}+B_{2}^{j} \cos \theta_{2}^{j}-C_{i j} \cos \theta_{1}^{i} \cos \theta_{2}^{j}\right)
$$

From Eq. 3.2 single differential cross sections can be derived in order to measure the polarization and the spin correlation of the $\bar{t} \bar{t}$ system.

$$
\begin{aligned}
& \frac{1}{\sigma} \frac{d \sigma}{d \cos \theta_{1}^{i}}=\frac{1}{2}\left(1+B_{1}^{i} \cos \theta_{1}^{i}\right) \quad \frac{1}{\sigma} \frac{d \sigma}{d \cos \theta_{2}^{j}}=\frac{1}{2}\left(1+B_{2}^{j} \cos \theta_{2}^{j}\right) \\
& \frac{1}{\sigma} \frac{d \sigma}{d \cos \theta_{1}^{i} \cos \theta_{2}^{j}}=\frac{1}{2}\left(1-C_{i j} \cos \theta_{1}^{i} \cos \theta_{2}^{j}\right) \ln \left(\frac{1}{\cos \theta_{1}^{i} \cos \theta_{2}^{j}}\right)
\end{aligned}
$$

\subsection{Measurement of the top quark polarization}

The di-lepton channel is perfect for a spin measurement because the spin analysing power of the leptons is close to one [6]. A kinematic fit is used to reconstruct the event. A $\chi^{2}$ minimization 
technique is used to unfold the distributions and 15 single differential cross sections are analysed in order to measure the polarization and the spin correlation factors. Figure 4 reports the results obtained compared to the SM predictions from different MC predictions.
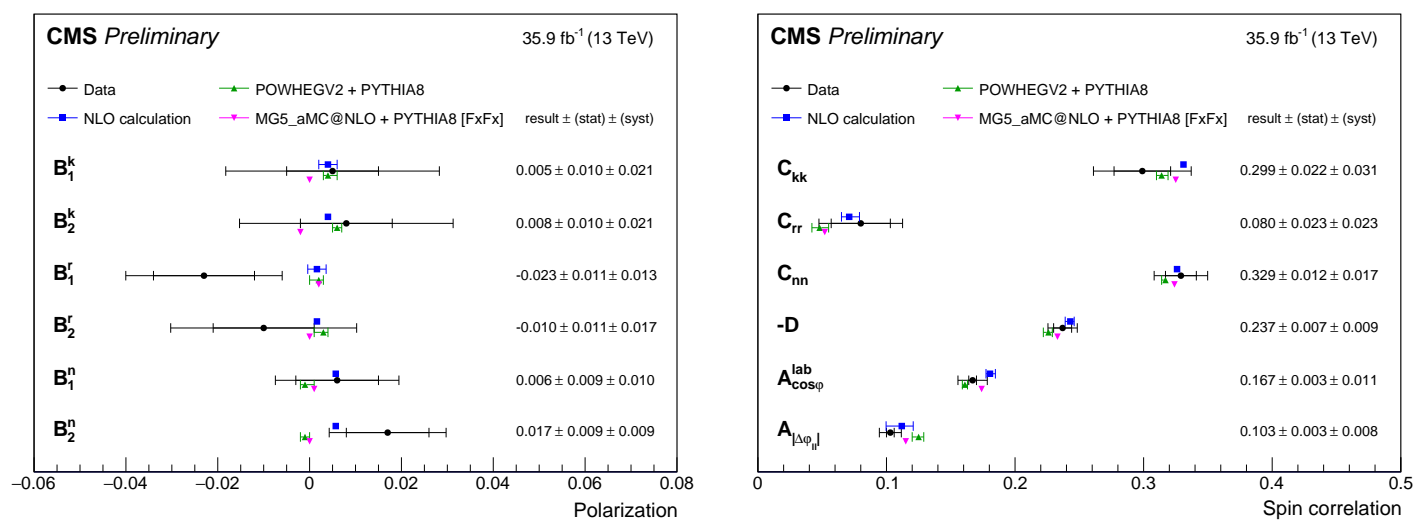

Figure 4: Polarization and the spin correlation factors compared to the SM predictions [6].

\section{Yukawa coupling}

Weak force mediated corrections affect the $\bar{t} \bar{t}$ cross section at loop-induced order $\alpha^{2} \alpha_{\text {weak }}$ and these become important only in particular kinematic regions with small relative velocity between the top quark and antiquark. The analysis is performed by requiring 1 high $p_{\mathrm{T}}$ isolated lepton, at least three jets, at least two of them are required to come from a $b$ quark [7]. A new kinematic technique has been developed to reconstruct events with on missing jet, that correspond to the low $\bar{t} \bar{t}$ system mass, $\mathrm{m}_{\mathrm{t}}$, region, the most sensitive to the Yukawa coupling. The extraction is made by comparing the data yields in $\mathrm{m}_{\mathrm{t}}$ and the difference of rapidity between top and antitop quarks for different number of reconstructed jets with distributions representing different Yukawa couplings. The results are given in terms of the signal strength $Y_{t}$, i.e. the ratio of the measured value over the expected one, and they are reported in Table 2.

\begin{tabular}{lcc}
\hline Channel & Expected 95\% CL & Observed 95\% CL \\
\hline 3 jets & $Y_{\mathrm{t}}<2.17$ & $Y_{\mathrm{t}}<2.59$ \\
4 jets & $Y_{\mathrm{t}}<1.88$ & $Y_{\mathrm{t}}<1.77$ \\
5 jets & $Y_{\mathrm{t}}<2.03$ & $Y_{\mathrm{t}}<2.23$ \\
\hline Combined & $Y_{\mathrm{t}}<1.62$ & $Y_{\mathrm{t}}<1.67$ \\
\hline
\end{tabular}

Table 2: Signal strengths for the Yukawa coupling in the different channels and in the combined one.

\section{Top quark spin asymmetry}

At the LHC the top quark can be produced singly via EW interactions. Due to the $V-A$ structure of the weak interaction the top quark is produced highly polarised along the spectator 
quark direction. A powerful observable to investigate the coupling structure in $t$-channel production is the differential cross section in terms of the polarization angle defined as the angle between the direction of the lepton and of the spectator quark in the top quark rest frame. The analysis requires a single isolated lepton, 2 or 3 quarks, up to 2 of them coming from a b quark [8]. The result obtained is $A_{e / \mu}=0.439 \pm 0.032$ (stat) \pm 0.053 (syst) and it is in agreement with the SM prediction.

\section{Conclusions}

Many properties of the top quark have been measured with high precision. Top quark mass is one of the most important: both direct and indirect measurements have uncertainties below $1 \mathrm{GeV}$.

The large amount of data collected by the CMS experiment allows measurement of rare processes to test the SM predictions. Many Beyond SM (BSM) scenarios can also be tested with differential and multi-differential measurements.

No deviations from the SM predictions are observed but the top quark sector is one of the most interesting for BSM manifestation.

\section{References}

[1] G. Corcella, "The top-quark mass: challenges in definition and determination," Front. in Phys. 7, 54 (2019) doi:10.3389/fphy.2019.00054 [arXiv:1903.06574 [hep-ph]].

[2] A. M. Sirunyan et al. [CMS Collaboration], "Measurement of the top quark mass with lepton+jets final states using p p collisions at $\sqrt{s}=13 \mathrm{TeV}$," Eur. Phys. J. C 78, no. 11, 891 (2018) doi:10.1140/epjc/s10052-018-6332-9 [arXiv:1805.01428 [hep-ex]].

[3] A. M. Sirunyan et al. [CMS Collaboration], "Measurement of the top quark mass in the all-jets final state at $\sqrt{s}=13 \mathrm{TeV}$ and combination with the lepton+jets channel," Eur. Phys. J. C 79, no. 4, 313 (2019) doi:10.1140/epjc/s10052-019-6788-2 [arXiv:1812.10534 [hep-ex]].

[4] A. M. Sirunyan et al. [CMS Collaboration], "Measurement of ț̄ normalised multi-differential cross sections in pp collisions at $\sqrt{s}=13 \mathrm{TeV}$, and simultaneous determination of the strong coupling strength, top quark pole mass, and parton distribution functions," [arXiv:1904.05237 [hep-ex]].

[5] A. M. Sirunyan et al. [CMS Collaboration], "Measurement of the tt production cross section, the top quark mass, and the strong coupling constant using dilepton events in pp collisions at $\sqrt{s}=13 \mathrm{TeV}$," Eur. Phys. J. C 79, no. 5, 368 (2019) doi:10.1140/epjc/s10052-019-6863-8 [arXiv:1812.10505 [hep-ex]].

[6] A. M. Sirunyan et al. [CMS Collaboration], "Measurement of the top quark polarization and $t \bar{t}$ spin correlations using dilepton final states in proton-proton collisions at $\sqrt{s}=13 \mathrm{TeV}$," arXiv:1907.03729 [hep-ex].

[7] A. M. Sirunyan et al. [CMS Collaboration], "Measurement of the top quark Yukawa coupling from $t \bar{t}$ kinematic distributions in the lepton+jets final state in proton-proton collisions at $\sqrt{s}=13 \mathrm{TeV}$," arXiv:1907.01590 [hep-ex].

[8] A. M. Sirunyan et al. [CMS Collaboration], "Measurement of differential cross sections and charge ratios for $t$-channel single top quark production in proton-proton collisions at $\sqrt{s}=13 \mathrm{TeV}$," arXiv:1907.08330 [hep-ex]. 